\title{
Numerical Investigation on the Thermodynamic Behaviour of the Friction Pairs in a Multi-disc Clutch
}

\author{
Biao Ma ${ }^{1}$, Liang Yu ${ }^{1}$, Man Chen' ${ }^{1}$ Jikai Liu², Jiaqi Xue ${ }^{1}$ \\ ${ }^{1}$ School of Mechanical Engineering, Beijing Institute of Technology \\ Beijing, 100081, P.R. China \\ mabiao@bit.edu.cn; yuliang0516@126.com; turb911@ bit.edu.cn; 264330785@qq.com \\ ${ }^{2}$ Institute of Microelectronics of Chinese Academy of Sciences \\ Beijing, 100029, P.R. China \\ $812361107 @ q q . c o m$
}

\begin{abstract}
The dynamic model of a multi-disc clutch is developed that can calculate the contact pressure and the spline friction force acting at each friction surface. The thermal model is employed to investigate the temperature variation of each friction component. The simulation results show that due to the existence of spline friction, the contact pressure decreases along the axial direction, thus leading to the decline of the spline friction force. Moreover, because of the attenuation of contact pressure, the surface temperature and friction torque decrease gradually along the axial direction. The highest temperature and friction torque both occur in the plate near the clutch piston. Therefore, the plate close to the piston is more prone to the thermal buckling.
\end{abstract}

Keywords: Multi-disc clutches, Spline friction, Contact pressure, Surface temperature, Friction torque.

\section{Nomenclature}
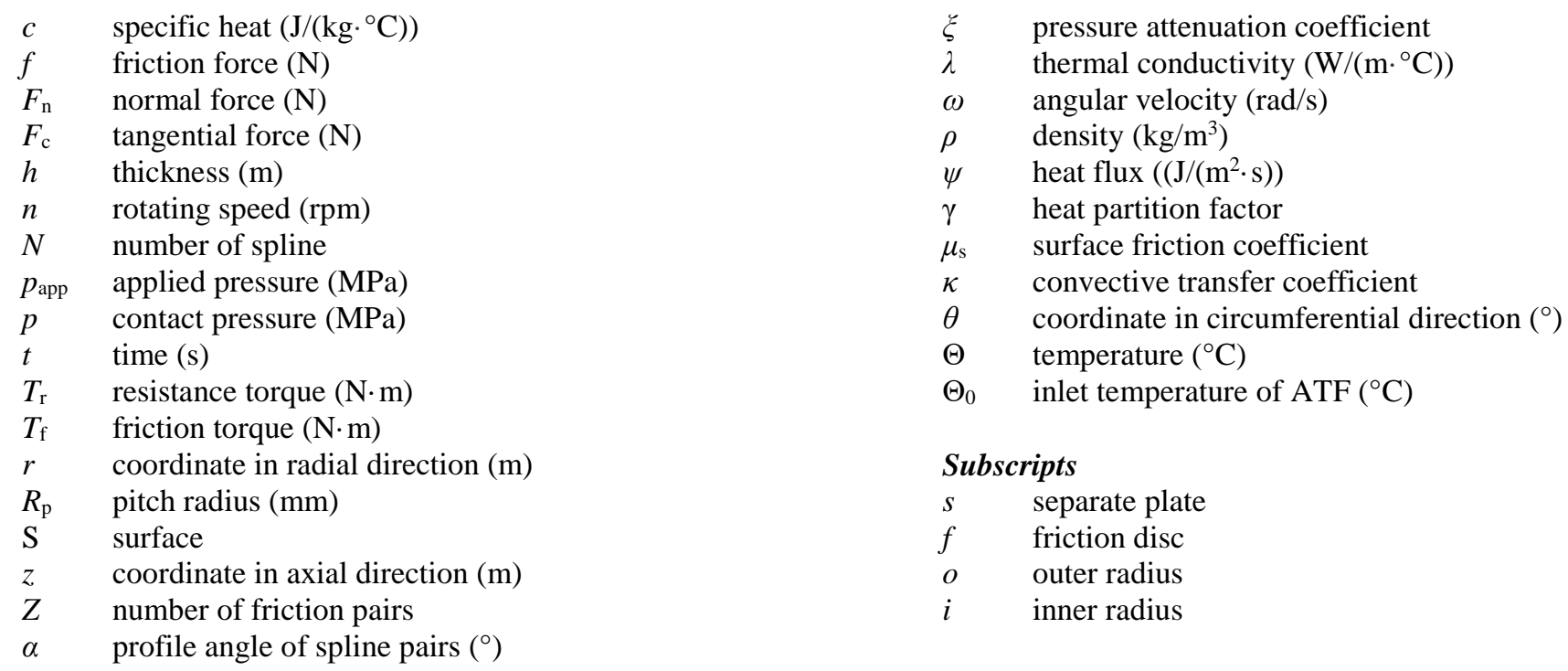

\section{Introduction}

The multi-disc clutch is one of the most important components in the transmission system [1]. In the multi-disc clutch pack, the friction discs are mounted to the driving gear by splines, and the separate plates splined to the cylinder liner to transfer power to the transmission system. The large amount of friction heat generated during the sliding period has been a lasting challenge which faced by the clutch designers and manufacturers [2]. So far, numerous researches have been conducted to analyse the transient thermal behaviour of the multi-disc clutch. 
Choi and Lee [3] investigated the transient thermodynamic behaviour of the brakes, indicating that the rotating speed had a great influence on the thermal state and contact ratio of the contact surfaces. Li et al. [4] studied the temperature field of the carbon fabric wet clutch during the engagement process by the finite element analysis. The maximum temperature distribution in a braking system was obtained with the thermal sensitivity of the disc material and the roughness of contact surface taken into account [5]. Yang et al. [6] investigated the thermal deformation and the contact pressure distribution on the friction surfaces, indicating that the contact pressures were different in different friction surfaces. In addition, Yu et al. [7] studied the generation and variation mechanism of the friction torque with the applied pressure, rotating speed and automatic transmission fluid (ATF) temperature taken into consideration. Marklund et al. [8] developed a torque transfer model in the boundary lubrication conditions.

However, the spline friction has a great influence on the attenuation of contact pressure along the axial direction. When the piston was moving along the axial direction at the early clutch engagement, the spline teeth of the friction components were subjected to the dynamic friction. After the gaps among the friction pairs were eliminated, the friction components still tended to move axially due to the existence of the applied pressure, so the dynamic friction of the spline teeth was converted to static friction. Surprisingly, there are relatively few studies devoted to the investigation of the spline friction. Newcomb et al. [9] established the axial force model of the friction surfaces with the consideration of spline force. A mathematical analysis was conducted to analyse the effects of the spline friction on the friction torque [10]. Now, this paper is conducted to study the thermodynamic performance of friction pairs in a multi-disc clutch with the consideration of spline friction.

\section{Numerical model}

\subsection{Dynamic model}

As shown in Fig.1, the friction components are numbered as $0,1,2, \cdots, i, i+1, \cdots, \mathrm{M}$, and the contact surfaces are also numbered as $S_{1}, S_{2}, \cdots, S_{M}$. To exemplify it, the plate 2 has two friction surfaces, namely $S_{2}$ and $S_{3}$. Moreover, the applied pressure $p_{\text {app }}$ is uniformly distributed on the piston.

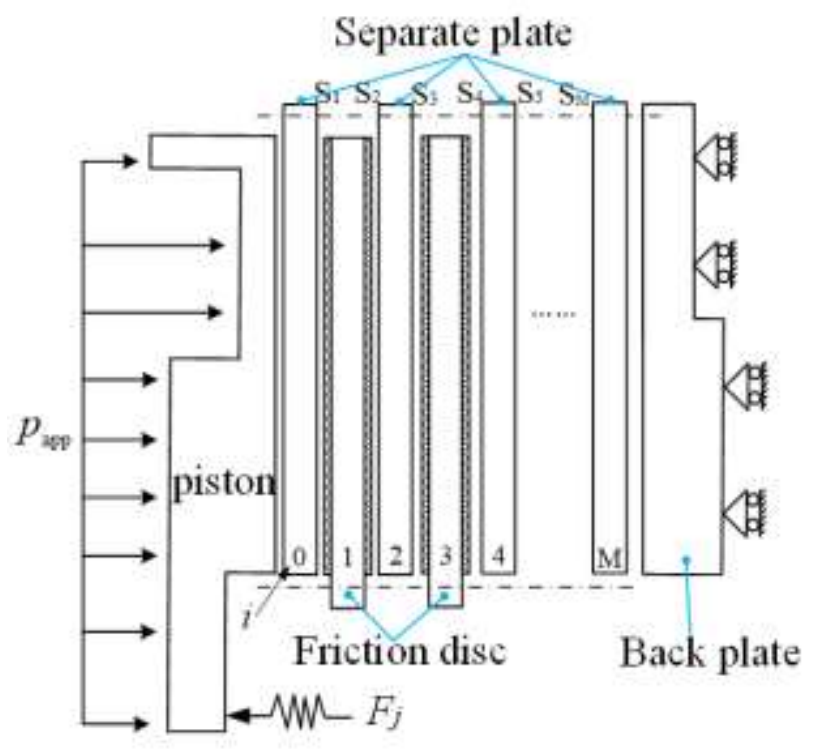

Fig. 1: Simplified 2D model of the multi-disc clutch. 


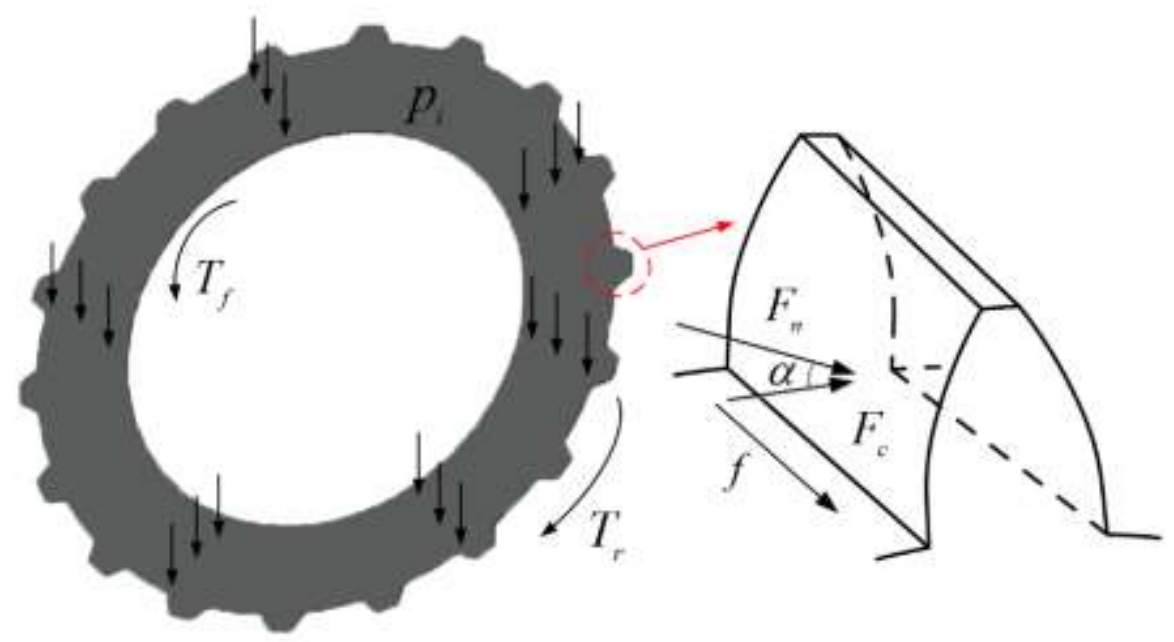

Fig. 2: Force diagram of friction components.

During the clutch engagement process, the forces on a single separate plate is shown in Fig. 2. The spline friction force can be deduced as

$$
f=\mu_{\mathrm{f}} N \cdot F_{\mathrm{n}}=\frac{\mu_{\mathrm{f}} T_{\mathrm{r}}}{R_{\mathrm{p}} \cos \alpha}
$$

where $\mu_{\mathrm{f}}$ is the spline coefficient of friction $(\mathrm{COF}), \mu_{\mathrm{f}}=0.12$ [11].

The contact pressure on each friction surface can be expressed as

$$
p_{i+1}=p_{i}-\frac{f}{A}
$$

Consequently, the friction torque on each separate plate can be given as

$$
T_{\mathrm{f}, i}=\frac{2 \pi \mu_{\mathrm{s}}\left(r_{o}^{3}-r_{i}^{3}\right)}{3} \times \begin{cases}p_{i+1} & i=0 \\ \left(p_{i}+p_{i+1}\right) & i=1,2,3, \ldots, \mathrm{M}-1 \\ p_{i} & i=\mathrm{M}\end{cases}
$$

The attenuation coefficient of contact pressure $\xi$ is introduced to characterize the contact pressure distribution among the friction surfaces.

$$
\xi=\frac{2 \mu_{\mathrm{s}} \mu_{\mathrm{f}}\left(r_{o}^{3}-r_{i}^{3}\right)}{3 R_{\mathrm{p}}\left(r_{o}^{2}-r_{i}^{2}\right) \cos \alpha}
$$

According to Eqs. (1-4), the friction force can be simplified as follows [10]. 


$$
f=A \xi \times \begin{cases}p_{i+1} & i=0 \\ \left(p_{i}+p_{i+1}\right) & i=1,2,3 \ldots \mathrm{M}-1 \\ p_{i} & i=\mathrm{M}\end{cases}
$$

Integrating Eqs. (1), (2) and (5), the contact pressure can be expressed as

$$
p_{i+1}= \begin{cases}\frac{1}{1+\xi} p_{i} & i=0 \\ \frac{1-\xi}{1+\xi} p_{i} & i=1,2,3 \ldots \mathrm{M}-1\end{cases}
$$

\subsection{Thermal model}

The heat conduction equation of the friction components can be written as

$$
\rho c \frac{\partial \Theta}{\partial t}=\frac{1}{r} \frac{\partial}{\partial r}\left(\lambda r \frac{\partial \Theta}{\partial r}\right)+\frac{\partial}{\partial z}\left(\lambda \frac{\partial \Theta}{\partial z}\right)
$$

It is assumed that the frictional work is totally converted into friction heat without any loss and the friction surfaces are in good contact. The friction heat on the friction pair is described as [12]

$$
\psi=\psi_{\mathrm{s}}+\psi_{\mathrm{f}}=\mu \cdot p \cdot \omega \cdot r
$$

where the surface COF are obtained by the pin-on-disc test [13]

$$
\mu_{\mathrm{s}}=23 e^{\left(\frac{-2.6 w}{(\ln \Theta+1)\left((28.3 p)^{0.4}-0.87\right)}-5.16\right)}-\frac{0.01 \ln (4 w+1)}{e^{0.005 \Theta}}+0.08\left(e^{-0.005 \Theta}-1\right)\left(e^{-0.2 w}-1\right)-0.005 \ln (28.3 p)+0.015
$$

According to the material characteristics of the plate and disc, the heat partition factor of $\mathrm{Cu}$-based friction pairs can be written as [14]

$$
\gamma=\frac{\sqrt{\lambda_{s} \rho_{s} c_{s}}}{\sqrt{\lambda_{s} \rho_{s} c_{s}}+\sqrt{\lambda_{f} \rho_{f} c_{f}}}
$$

Therefore, the heat flux, conducting to the plate and disc, equals:

$$
\begin{gathered}
\psi_{s}=\gamma \psi \\
\psi_{f}=(1-\gamma) \psi
\end{gathered}
$$


Additionally, the boundary conditions are applied as follows.

$$
\begin{gathered}
\left.\lambda \frac{\partial \Theta(r, \mathrm{z}, t)}{\partial r}\right|_{r=r_{i}}=+\kappa_{i}\left[\Theta(r, \mathrm{z}, t)-\Theta_{\text {oil }}\right], 0 \leq z \leq h \\
\left.\lambda \frac{\partial \Theta(r, \mathrm{z}, t)}{\partial r}\right|_{r=r_{o}}=-\kappa_{o}\left[\Theta(r, \mathrm{z}, t)-\Theta_{o i l}\right], 0 \leq z \leq h \\
\left.\lambda \frac{\partial \Theta(r, \mathrm{z}, t)}{\partial z}\right|_{\mathrm{z}=0}=\psi_{s, i}(r, \mathrm{z}, t), r_{i} \leq r \leq r_{o} \\
\left.\lambda \frac{\partial \Theta(r, \mathrm{z}, t)}{\partial z}\right|_{\mathrm{z}=H}=\psi_{s, i+1}(r, \mathrm{z}, t), r_{i} \leq r \leq r_{o} \\
\left.\Theta(r, z, t)\right|_{t=0}=\Theta_{0}
\end{gathered}
$$

\section{Results and discussion}

Considering the spine friction, the thermodynamic characteristics of a 16-friction-pair clutch system are discussed in this section. The simulation conditions are as follows. The initial rotating speed is 1500rpm and it decreases linearly; the applied pressure keeps at $1 \mathrm{MPa}$. The engagement process lasts 1 second. Additionally, the separate plate is made of $65 \mathrm{Mn}$ steel; the friction disc is coated by the copper based powder metallurgy. The inner and outer radii of the friction components are $0.060 \mathrm{~m}$ and $0.073 \mathrm{~m}$, respectively. The material parameters of the friction components are listed in Table 1.

Table 1: Overview of the material parameters.

\begin{tabular}{lccc}
\hline Parameters & Thermal conductivity $\mathrm{W} /\left(\mathrm{m} \cdot{ }^{\circ} \mathrm{C}\right)$ & Density $\left(\mathrm{kg} / \mathrm{m}^{3}\right)$ & Specific heat $\mathrm{J} /\left(\mathrm{kg} \cdot{ }^{\circ} \mathrm{C}\right)$ \\
\hline Separate plate & 45.90 & 7800 & 487 \\
Friction disc & 9.30 & 5500 & 460 \\
\hline
\end{tabular}




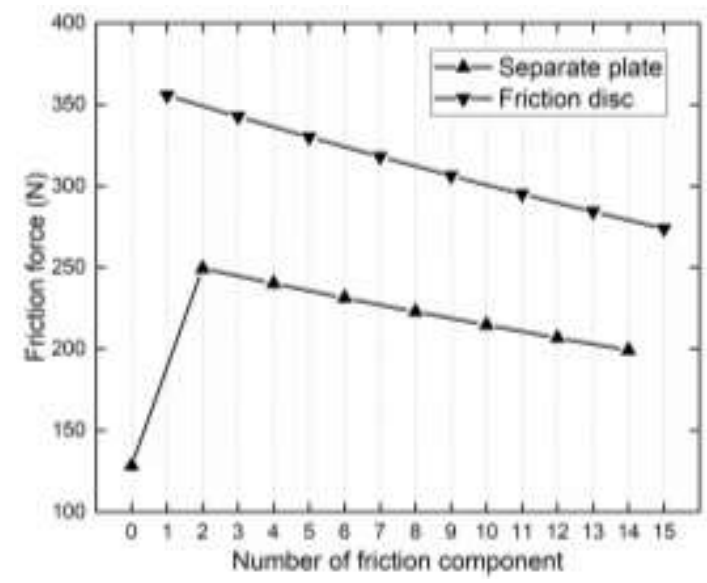

(a) Spline friction force

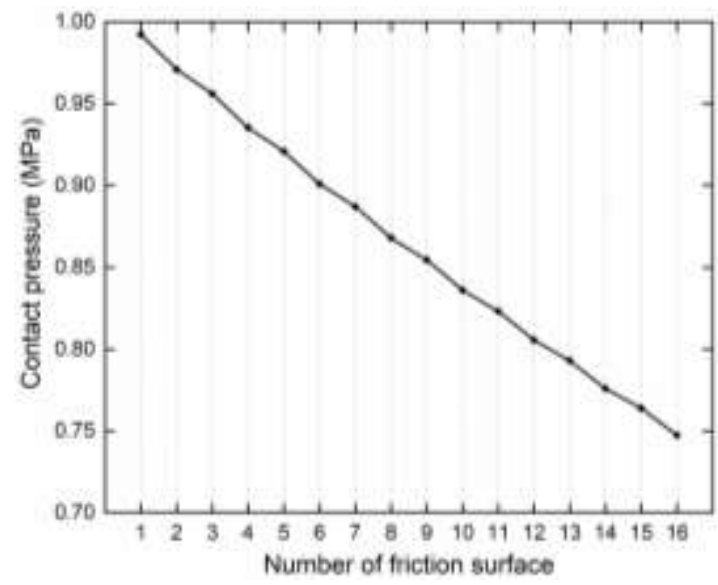

(b) Contact pressure

Fig. 3: Variations of the spline friction force and contact pressure along the axial direction.

Figure 3 demonstrates the variations of the spline friction force and contact pressure along the axial direction in a 16friction-pair system. As shown in Fig. 3(a), the maximum friction force appears in the disc 1 which is $377.6 \mathrm{~N}$; due to the fact that Plate 0 has only one surface to transfer the friction torque, the minimum friction force appears in Plate 0 and the value is $128.5 \mathrm{~N}$. Apart from Plate 0, the spline friction force of plate decreases linearly with the increase of the friction components, so does the friction force of disc. In addition, as shown in Eq. (1), since the pitch radius of friction disc is smaller than that of separate plate, the spline friction force of disc is much larger than that of separate plate. As shown in Fig. 3(b), the contact pressure decreases almost linearly along the axial direction. To be more specific, the contact pressure on the surface $S_{16}$ is only $0.75 \mathrm{MPa}$, implying that the contact pressure decreases by $0.25 \mathrm{MPa}$ from $S_{1}$ to $S_{16}$. However, the spline force, which can bring about the decrease of contact pressure, not only affects the torque transfer capacity of the clutch, but also have a great influence on the temperature field of the clutch.

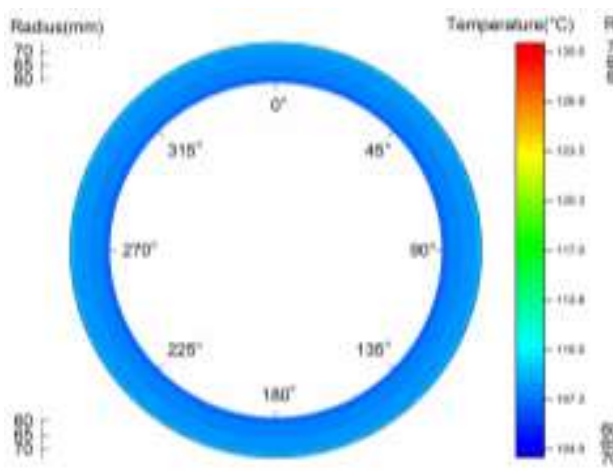

(a)

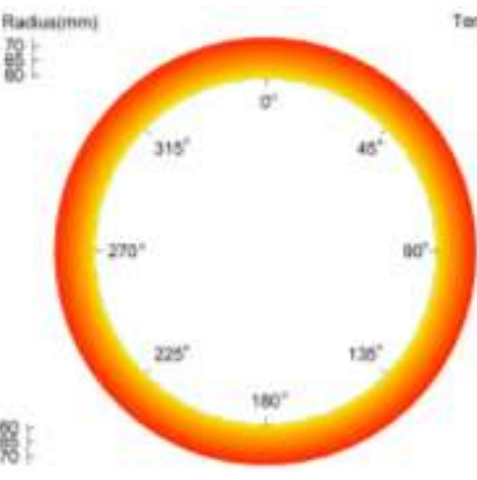

(b)
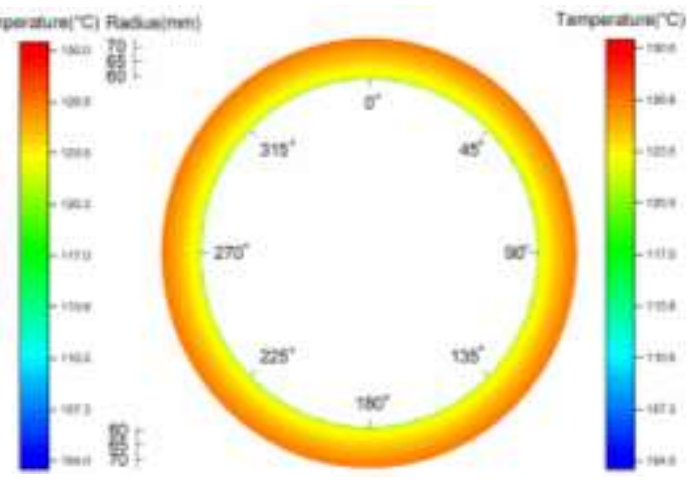

(c) 

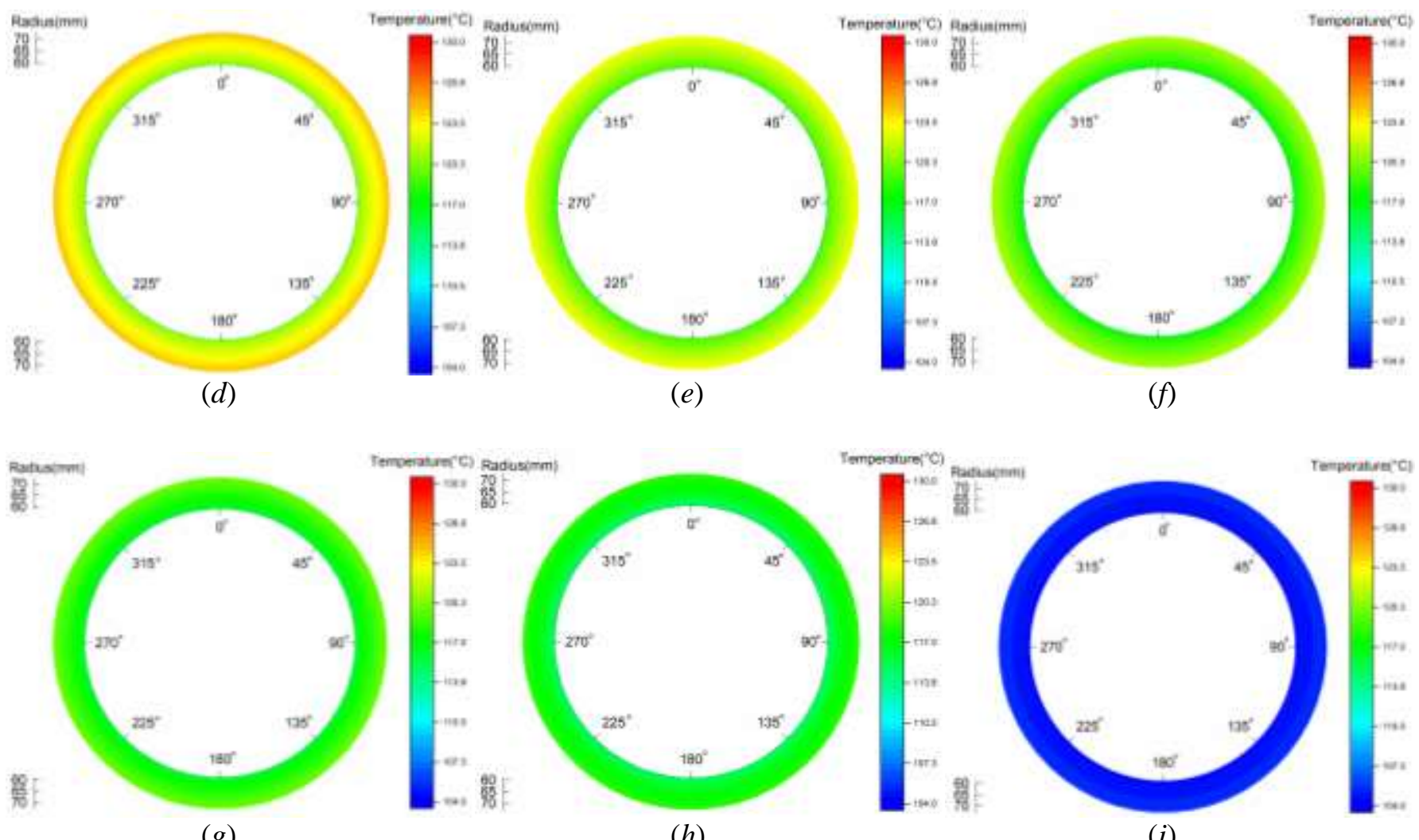

$(g)$

$(h)$

(i)

Fig. 4: Radial temperature field of the separate plates. (a)- (i): Plate 0-16.

Figure 4 depicts the radial temperature field of the 16-friction-pair cutch system at the end of the engagement. It should be noted that the Plate 0 and Plate 16 just have one friction surface to produce the heat flux, while there is a dramatic thermal diffusion on the other surface of both plates. Consequently, the temperature in these two plates is much lower than that of other plates. Apart from that, the surface temperature decreases gradually along the axial direction. That can be interpreted as follows. It is known that the heat flux is proportional to the contact pressure which decreases along the axial direction because of the spline friction as shown in Fig.3 (b). As a result, there exists a significant temperature gradient along the axial direction, and the highest temperature occurs in the plate 2 which is $128.9^{\circ} \mathrm{C}$. In addition, the plate and disc close to the piston are more prone to thermal buckling and thermal degradation. 


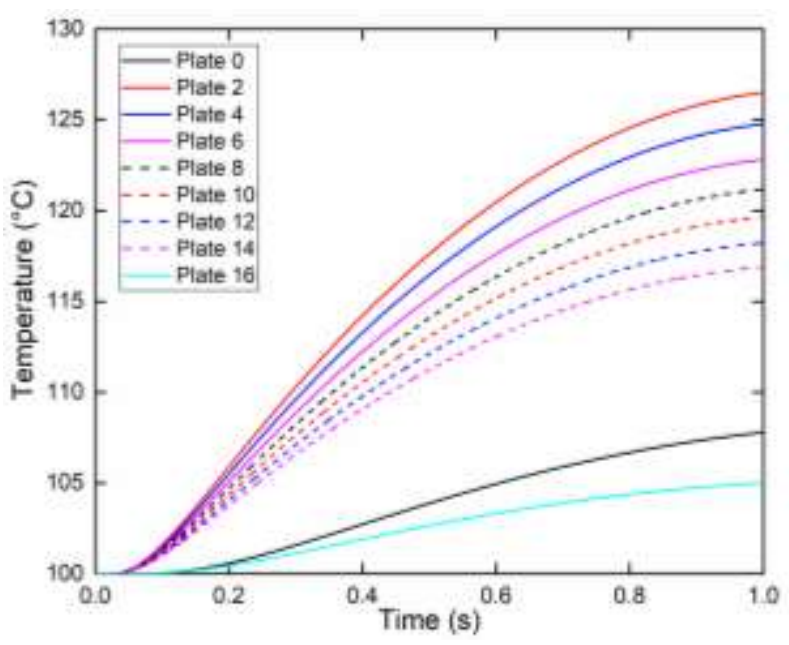

(a) Average temperature

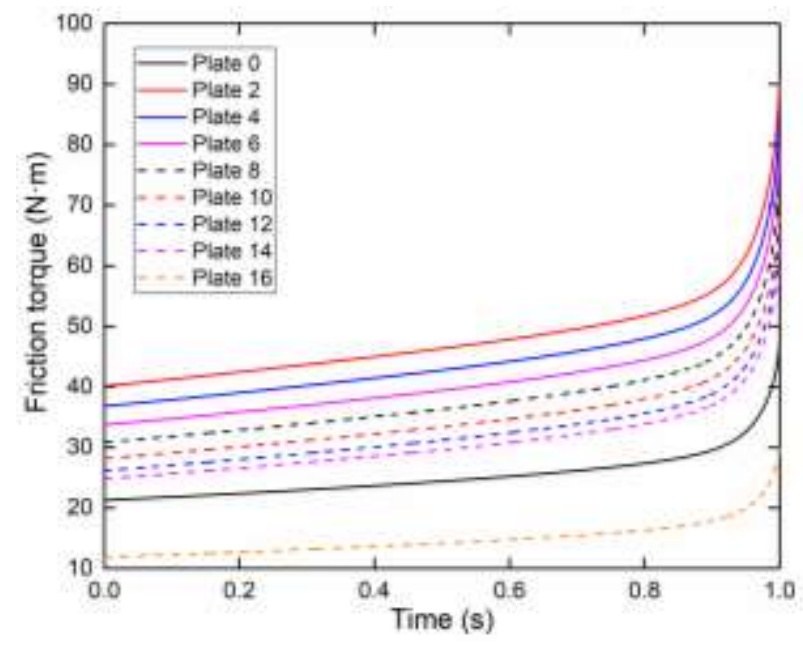

(b) Friction torque

Fig. 5: Variations of the average temperature and friction torque among the friction components.

Figure 5 demonstrates the variations of the average temperature and friction torque during the engagement process. Because the radial temperature of plate increases linearly as shown in Fig.4, the temperature in the medial radius can be regarded as the average temperature. It should be noted that both the average temperature and friction torque decreases along the axial direction. The reason is that the contact pressure is also proportional to the friction torque. The friction torque decreases from $90.3 \mathrm{~N} \cdot \mathrm{m}$ to $62.4 \mathrm{~N} \cdot \mathrm{m}$ at the early engagement from the Plate 2 to Plate 14; The friction torques of Plate 0 and Plate 16 are respectively $47.3 \mathrm{~N} \cdot \mathrm{m}$ and $30.0 \mathrm{~N} \cdot \mathrm{m}$, implying that the friction torque decrease by $17.3 \mathrm{~N} \cdot \mathrm{m}$ from $\mathrm{S}_{1}$ to $\mathrm{S}_{16}$. It is usually assumed that the total friction torque of a multi-disc clutch will merely be the friction torque on a single separate plate multiplied by the number of such plates. The existence of the spline force means that it is clearly impossible. The growth rate of the friction torque decreases with the increase of friction components.

\section{Conclusion}

The thermodynamic analysis of a 16-friction-pair clutch system has been performed to investigate the effect of the spline friction on the variations of the temperature and friction torque. The simulation results demonstrated that the contact pressure decreased linearly along the axial direction because of the spline friction, thus leading to the decline of the spline friction force. However, due to the fact that the pitch radius of disc is smaller than that of plate, the spline friction force of disc is much larger than that of plate. Moreover, because of the attenuation of contact pressure, the surface temperature and friction torque decrease gradually along the axial direction. The highest temperature and friction torque both occurred in the Plate 2 . As a result, the plate and disc close to the piston were more prone to the thermal buckling and thermal degradation. The friction torque increased nonlinearly with the increase of friction pairs that the calculation of friction torque should consider the attenuation of contact pressure.

\section{Acknowledgements}

This work was supported by the National Natural Science Foundation of China (Grant NO. 51775045 and NO. 51575042). 


\section{References}

[1] G. Li, D. Görges, "Optimal control of the gear shifting process for shift smoothness in dual-clutch transmissions," Mechanical Systems and Signal Processing, vol. 103, pp. 23-38, 2018.

[2] L. Yu, B. Ma, H. Li, L. Jikai, L. Mingyang, "Numerical and Experimental Studies of a Wet Multi-disc Clutch on Temperature and Stress Fields Excited by the Concentrated Load," Tribology Transactions, pp. 1-49, 2018.

[3] J. Choi, I. Lee, "Transient thermoelastic analysis of disk brakes in frictional contact," Journal of Thermal Stresses, vol. 26, no. 3, pp. 223-244, 2003.

[4] L. Wenbin, H. Jianfeng, F. Jie, C. Liyun, Y. Chunyan, "Simulation and application of temperature field of carbon fabric wet clutch during engagement based on finite element analysis," International Communications in Heat and Mass Transfer, vol. 71, pp. 180-187, 2016.

[5] A. Yevtushenko, P. Grzes, "Maximum temperature in a three-disc thermally nonlinear braking system," International Communications in Heat and Mass Transfer, vol. 68, pp. 291-298, 2015.

[6] L. Yang, B. Ma, M. Ahmadian, L. Heyan, B. Vick, "Pressure Distribution of a Multidisc Clutch Suffering Frictionally Induced Thermal Load," Tribology Transactions, vol. 59, no. 6, pp. 983-992, 2016.

[7] L. Yu, B. Ma, M. Chen, L. Heyan, J. Liu, L. Zheng, "Numerical and experimental studies on the characteristics of friction torque based on wet paper-based clutches," Tribology International, 2018.

[8] P. Marklund, R. Mäki, R. Larsson, E. Höglund, "Thermal influence on torque transfer of wet clutches in limited slip differential applications," Tribology international, vol. 40, no. 5, pp. 876-884, 2007.

[9] T. P. Newcomb, H. E. Merritt, "Effect of Spline Friction on the Torque Capacity and Interface Temperatures Reached during a Multi-Disc Clutch Engagement," Journal of Mechanical Engineering Science, vol. 4, no. 4, pp. 353-355, 1962.

[10] E. F. Finkin, "The Consequences of Spline Friction in Multiple Disk Brake and Clutch Packs," Journal of Lubrication Technology, vol. 90, no. 1, pp. 65-71, 1968.

[11] J. Cui, C. Wang, F. Xie, R. Xuan, G. Shen, "Numerical investigation on transient thermal behavior of multidisk friction pairs in hydro-viscous drive," Applied Thermal Engineering, vol. 67, no. 1-2, pp. 409-422, 2014.

[12] D. Meresse, S. Harmand, M. Siroux, M. Watremez, L. Dubar, "Experimental disc heat flux identification on a reduced scale braking system using the inverse heat conduction method," Applied Thermal Engineering, vol. 48, pp. 202-210, 2012.

[13] E. Zhao, B. Ma, H. Li, "Numerical and Experimental Studies on Tribological Behaviors of Cu-Based Friction Pairs from Hydrodynamic to Boundary Lubrication," Tribology Transactions, vol. 61, no. 2, pp. 347-356, 2018.

[14] A. Yevtushenko, P. Grzes, "Finite element analysis of heat partition in a pad/disc brake system," Numerical Heat Transfer, Part A: Applications, vol. 59, no. 7, pp. 521-542, 2011. 